02.1;09.2

\title{
Фазовый переключатель для электромагнитно индуцированных решеток в среде с $\Lambda$-атомами
}

\author{
() М.Ю. Гордеев, Ю.В. Рождественский
}

${ }^{1}$ Санкт-Петербургский национальный исследовательский университет информационных технологий, механики и оптики (Университет ИТМО), Санкт-Петербург, Россия

E-mail: mxmgordeev@gmail.com

Поступило в Редакцию 19 марта 2019г.

В окончательной редакции 19 марта 2019 г.

Принято к публикации 21 марта 2019г.

\begin{abstract}
Теоретически исследована возможность использования фазового эффекта выключения когерентного пленения населенностей для создания переключателя направления распространения света в электромагнитно индуцированных решетках в среде с $\Lambda$-атомами. Определена допустимая область применения эффекта, получены и исследованы параметры, позволяющие получить высокоэффективную схему пространственного перераспределения интенсивности пробного поля на решетках атомной плотности.
\end{abstract}

Ключевые слова: электромагнитно индуцированные решетки, оптические устройства, оптический переключатель, оптический маршрутизатор.

DOI: 10.21883/PJTF.2019.12.47910.17793

В последнее время большое внимание уделяется такому квантовому оптическому эффекту, как электромагнитно индуцированные решетки (ЭМИР). Пионерские исследования были посвящены трехуровневой $\Lambda$-схеме $[1,2]$. В результате дальнейших исследований по этой тематике были получены различные показатели эффективности перераспределения интенсивности в максимумы первого порядка, предложены различные способы увеличения эффективности $[3,4]$. Одним из основных способов практического применения эффекта является создание быстродействующего компактного оптического маршрутизатора $[5,6]$. Помимо высокой эффективности и компактности одной из важнейших его функций является возможность быстрого перенаправления света без остановки работы системы.

В настоящей работе предложен новый способ переключения направления света в ЭМИР с использованием фазового эффекта в замкнутой $\Lambda$-системе. Особенностью исследованной схемы является простота реализации и эксплуатации, а также высокая эффективность перераспределения интенсивности пробного поля в максимумы первого порядка.

Энергетическая схема атомных уровней в замкнутой $\Lambda$-конфигурации представлена на рис. $1, a$, схема моделируемого эксперимента приведена на рис. $1, b$. На переходе $|2>-| 3>$ атомной системы вдоль направления $O z$ действует поле пробной бегущей волны с частотой Раби $\Omega_{2}$ и отстройкой $\Delta_{2}$ от резонансного значения. Радиочастотное поле с частотой Раби $\Omega_{r}$ действует вдоль направления оси $O x$ на переходе $|1>-| 2>$ резонансно. Поля, действующие на переходе $|1>-| 3>$, направлены вдоль оси $O x$ и имеют круговую поляризацию в противоположных направлениях. На переходе $|1>-| 3>$ действует поле сильной стоячей волны с пространственно- зависимой частотой Раби $\Omega_{1}(x)$ и отстройкой $\Delta_{1}$ от резонансного значения. Оптические релаксации по каналам $|3>-| n>(n=1,2)$ определяются константами $\gamma_{1}$ и $\gamma_{2}$ соответственно. Естественная ширина уровня $\mid 3>$ равна $2 \gamma=\gamma_{1}+\gamma_{2}$. Полуширины оптических переходов обозначены как $\Gamma_{13}$ и $\Gamma_{23}$. Помимо этого возможен распад когерентностей между нижними состояниями атома (релаксации низкочастотных когерентностей) со скоростью $\Gamma_{12}$.

Запишем поле, модулирующее атомы в среде, в виде

$$
\begin{aligned}
\mathbf{E}_{1} & =\mathbf{e}_{1}^{1} E_{1} \cos \left(\omega_{1} t-k_{1} x+\phi_{1}\right) \\
& +\mathbf{e}_{1}^{2} E_{1} \cos \left(\omega_{1} t+k_{1} x+\phi_{1}\right),
\end{aligned}
$$

в то время как поле пробной волны и радиочастотное поле имеют вид

$$
\begin{aligned}
& \mathbf{E}_{2}=\mathbf{e}_{2} E_{2} \cos \left(\omega_{2} t-k_{2} z+\phi_{2}\right), \\
& \mathbf{E}_{r}=\mathbf{e}_{r} E_{r} \cos \left(\omega_{r} t-k_{r} x+\phi_{r}\right) .
\end{aligned}
$$

Здесь волны с $\omega_{1}, k_{1}$ и $\omega_{r}, k_{r}$ распространяются вдоль оси $O x$, а пробная волна с $\omega_{2}, k_{2}$ распространяется в положительном направлении оси $O z ; \phi_{1}, \phi_{2}, \phi_{r}-$ фазы соответствующих волн. Следует отметить важность векторов $k_{1}, k_{2}, k_{r}$ в формулах (1), так как дальнейшее рассмотрение задачи пространственной дифракции не представляется возможным без них.

Воспользуемся для исследования системой самосогласованных уравнений, которая состоит из уравнений Лиувилля для элементов матрицы плотности в приближении вращающейся волны и дипольном приближении для описания состояния среды и укороченного волнового уравнения в приближении медленно меняющихся амплитуд для описания распространения пробного поля в среде. 

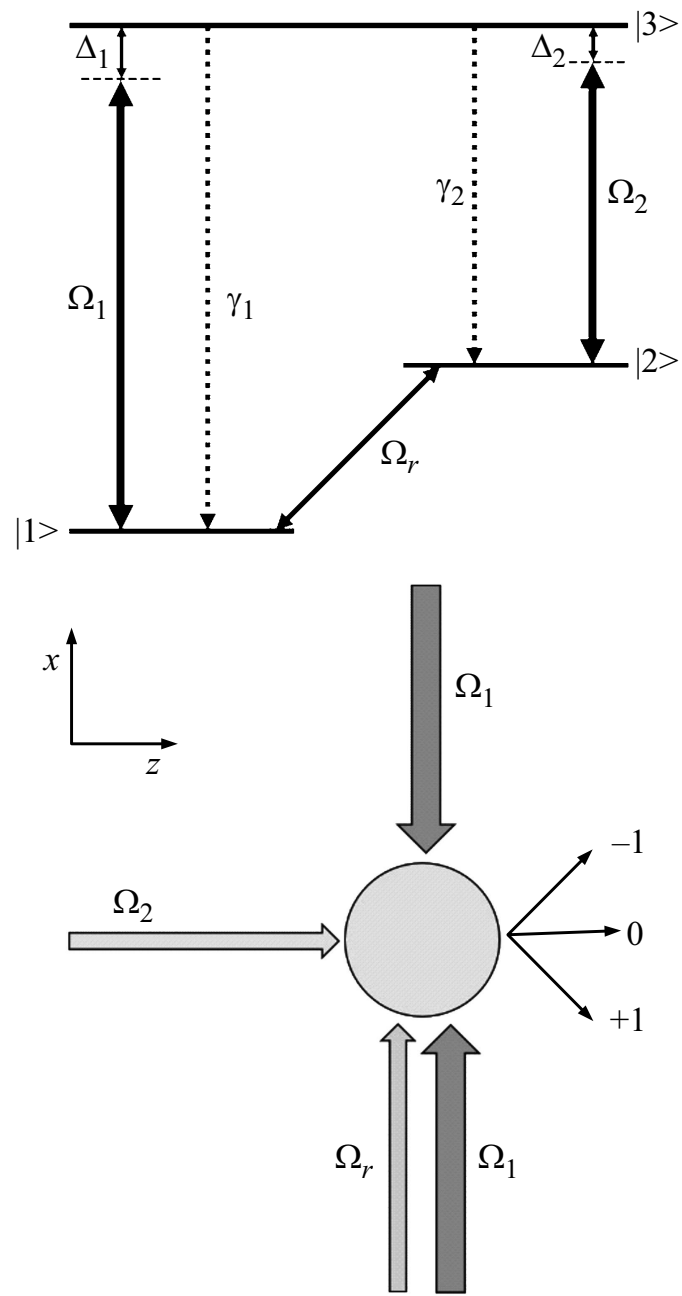

$b$

Рис. 1. Энергетическая схема атомных уровней в замкнутой $\Lambda$-конфигурации $(a)$ и принципиальная схема эксперимента (b).

Уравнение Лиувилля, описывающее динамику состояний атомов, имеет вид

$$
i \hbar \dot{\tilde{\rho}}_{i j}=[H, \tilde{\rho}]_{i j}+i \Gamma_{i j} \tilde{\rho}_{i j}
$$

с гамильтонианом взаимодействия в виде $H=H_{0}+V$, где $H_{0}$ задает внутреннее состояние системы без возмущения, а $V=-\left(\mathbf{d}_{13} \cdot \mathbf{e}_{1}\right) E_{1} / \hbar-\left(\mathbf{d}_{23} \cdot \mathbf{e}_{2}\right) E_{2} / \hbar-\left(\mathbf{d}_{12} \cdot \mathbf{e}_{r}\right) E_{r} / \hbar$ определяет взаимодействие атомов с полем оптического излучения и радиочастотным полем для переходов $|n>-| 3>(n=1,2)$ и $|1>-| 2>$ с матричным элементом оператора дипольного взаимодействия.

В выражении (2) матрица $\Gamma_{i j}$ задает скорости релаксации элементов $\tilde{\rho}_{i j}(x, t)$. При этом скорость релаксации диагональных матричных элементов (т.е. населенностей) определяется естественной шириной $2 \gamma$ верхнего возбужденного состояния системы (рис. 1,a): $2 \gamma=\gamma_{1}+\gamma_{2}$, а скорости релаксации недиагональных матричных элементов $\Gamma_{i j}(i \neq j)$ могут наряду со скоростью естественного распада учитывать и другие воз- можные механизмы уширения (столкновения, конечную ширину спектра возбуждающих полей и пр.).

С учетом обозначенных выше приближений уравнение Лиувилля (2) сводится к системе уравнений для элементов матрицы плотности $\rho_{i j}(x, y, t)$ трехуровневого атома следующего вида:

$$
\begin{gathered}
i \dot{\rho}_{11}=\Omega_{r}\left(\rho_{12}-\rho_{21}\right)+\Omega_{1}\left(\rho_{13}-\rho_{31}\right)+i \gamma \rho_{33} \\
i \dot{\rho}_{22}=\Omega_{r}\left(\rho_{21}-\rho_{12}\right)+\Omega_{2}\left(\rho_{23}-\rho_{32}\right)+i \gamma \rho_{33} \\
i \dot{\rho}_{33}=\Omega_{1}\left(\rho_{31}-\rho_{13}\right)+\Omega_{2}\left(\rho_{32}-\rho_{23}\right)-2 i \gamma \rho_{33} \\
i \dot{\rho}_{12}=-\left(\Delta_{1}-\Delta_{2}\right) \rho_{12}+\Omega_{r}\left(\rho_{11}-\rho_{22}\right) \\
-\Omega_{1} \rho_{32} e^{i \Phi}+\Omega_{2} \rho_{13} e^{i \Phi}-i \Gamma_{12} \rho_{12} \\
i \dot{\rho}_{13}=-\Delta_{1} \rho_{13}+\Omega_{1}\left(\rho_{11}-\rho_{33}\right) \\
-\Omega_{r} \rho_{23} e^{-i \Phi}+\Omega_{2} \rho_{12} e^{-i \Phi}-i \Gamma_{13} \rho_{13} \\
i \dot{\rho}_{23}=-\Delta_{2} \rho_{23}+\Omega_{2}\left(\rho_{22}-\rho_{33}\right) \\
-\Omega_{r} \rho_{13} e^{i \Phi}+\Omega_{1} \rho_{21} e^{i \Phi}-i \Gamma_{23} \rho_{23}
\end{gathered}
$$

где $\rho_{i j}=\rho_{j i}^{*}, \quad$ частоты Раби $\Omega_{n}=\left(\mathbf{d}_{n 3} \cdot \mathbf{e}_{n}\right) E_{n}$, $\Omega_{r}=\left(\mathbf{d}_{12} \cdot \mathbf{e}_{r}\right) E_{r} / 2 \hbar, \quad \Phi=\phi_{r}+\phi_{2}-\phi_{1}-$ суммарная фаза контура. Считаем, что система замкнута: $\rho_{11}+\rho_{22}+\rho_{33}=1$, будем также считать, что распадов низкочастотных когерентностей в системе нет (по сравнению с другими распадами системы $\Gamma_{12}=0$ ).

При выводе системы уравнений (3) мы пренебрегли членами, содержащими временны́е осцилляции на удвоенной оптической частоте (резонансное приближение), и использовали так называемое приближение вращающейся волны, которое состоит в пренебрежении быстро осциллирующими членами уравнения по сравнению с медленно осциллирующими.

Воспользуемся волновым уравнением Максвелла для описания распространения пробного поля в среде, которое в приближении медленно меняющихся амплитуд и в стационарном режиме сводится к следующему виду:

$$
-\frac{i}{2 k_{2}} \frac{\partial^{2} E_{02}}{\partial x^{2}}+\frac{\partial E_{02}}{\partial z}=i \frac{4 \pi k_{2}}{2 \varepsilon_{0}} P_{02}
$$

где $E_{02}-$ медленно меняющаяся амплитуда пробного поля, $\varepsilon_{0}$ - диэлектрическая проницаемость вакуума. Используя усреднение дипольного момента на ансамбле однородно уширенной среды, получаем выражение для поляризации среды в виде $P_{02}=2 N d_{23} \rho_{23}$, где $N-$ атомарная плотность, $d_{23}$ - проекция дипольного момента перехода $|2>-| 3>\mid$ на единичный вектор поляризации среды, $\rho_{23}$ - элемент матрицы плотности, когерентность перехода $|2>-| 3>$.

Для получения аналитических выражений вещественной и мнимой частей элемента $\rho_{23}$ воспользуемся стационарным решением уравнений (3), которое может быть получено в пренебрежении временны́ми производными в левых частях по сравнению с членами в правых частях, содержащих скорости распада. Отметим, что в 

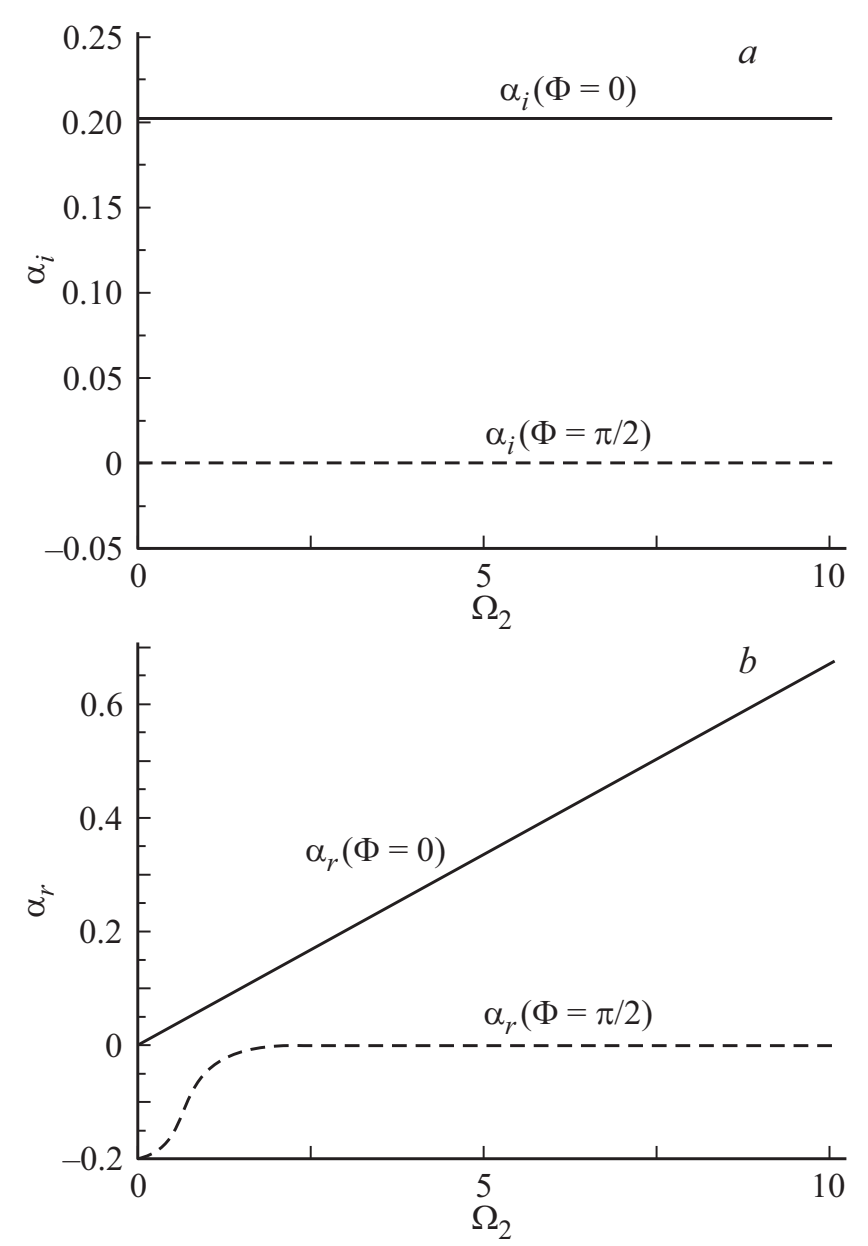

Рис. 2. Коэффициенты преломления $\alpha_{i}(a)$ и поглощения $\alpha_{r}(b)$ в зависимости от частоты Раби пробного поля $\Omega_{2}$. Остальные параметры системы следующие: $\Omega_{1}=3 \gamma, \Omega_{r}=1 \gamma$, $\Delta_{1}=\Delta_{2}=0$.

полученных выражениях учтены члены всех порядков для модулирующих $\Omega_{1}(x)$ и радиочастотного $\Omega_{r}$ полей, но только линейные члены для поля пробной волны $\Omega_{2}$.

Подставим это решение в уравнение (4) и заменим поляризацию в уравнении на получившееся после подстановки выражение. Для того чтобы получить окончательное выражение в безразмерной форме, выразим все распады, отстройки и частоты через $\gamma_{1}$, а координаты через $\Lambda_{x}$ для оси $O x$ и $z_{0}$ для оси $O z$, где $\Lambda_{x}=\pi / k_{x}-$ пространственный период наведенной электромагнитно индуцированной решетки, $k_{x}$ - волновой вектор сильных стоячих волн, а $z_{0}$ имеет вид $z_{0}=2 \hbar \gamma_{1} \varepsilon_{0} / N k_{2} d_{23}^{2}$. Тогда уравнение для поля приобретает вид

$$
-i \frac{\partial^{2} \Omega_{2}}{N_{F} \partial x^{2}}+\frac{\partial \Omega_{2}}{\partial z}=\left(\alpha_{r}+i \alpha_{i}\right) \Omega_{2},
$$

где $\alpha_{r}$ и $\alpha_{i}-$ коэффициенты поглощения и преломления пробного поля, а $N_{F}$ - число щелей ширины $2 \sqrt{\pi} \Lambda_{x}$, умещающихся на расстоянии $z_{0}: N_{F}=\left(2 \sqrt{\pi} \Lambda_{x}\right)^{2} / \lambda_{2} z_{0}$.

Решая получившееся уравнение для поля, находим поле на выходе из среды. Отношение поля на выходе
$\Omega_{2 o u t}$ и поля на входе $\Omega_{2 \text { in }}$ дает вид функции трансляции среды толщиной $L$ (по оси $O z$ ) по отношению к полю

$$
T(x)=\exp \left[\alpha_{r}(x) L\right] \exp \left[i \alpha_{i}(x) L\right]
$$

где $T(x)=\Omega_{2 o u t} / \Omega_{2 i n}, L-$ толщина среды вдоль оси $O z$.

Рассматривая вклад только дальнего поля (дифракция Фраунгофера) и считая, что волна пробного поля плоская и имеет одинаковую амплитуду поперек пучка ширины $M \Lambda_{x}$, определим интенсивность $I_{\text {out }}(\theta)$ как

$$
\begin{gathered}
I_{\text {out }}(\theta)=\left|\Omega_{2}^{1}(\theta)\right|^{2} \frac{\sin ^{2}\left(M \pi \Lambda_{x} \sin \theta / \lambda_{2}\right)}{M^{2} \sin ^{2}\left(\pi \Lambda_{x} \sin \theta / \lambda_{2}\right)}, \\
\Omega_{2}^{1}(\theta)=\int_{0}^{1} T(x) \exp \left[-i 2 \pi \Lambda_{x} x \sin \theta / \lambda_{2}\right] d x .
\end{gathered}
$$

Согласно [7], в случае резонансных отстроек в системе возникает эффект когерентного пленения населенностей (КПН), в результате чего вся населенность в системе оказывается сосредоточенной в суперпозиционном состоянии $|1>+| 2>$. В этом случае не возникает перераспределения интенсивности пробного поля.

Как видно, коэффициент преломления $\alpha_{i}$ не содержит линейной части по пробному полю (рис. 2, $a$, кривая $\Phi=0)$, ввиду чего не возникает ЭМИР и, как следствие, пробное поле не перенаправляется в максимумы первого порядка. Даже при выключении КПН путем изменения фазы создать ЭМИР при таких условиях в этой системе не представляется возможным, так как коэффициент преломления равен нулю (рис. $2, a$, кривая $\Phi=\pi / 2$ ).

Но при небольшом изменении отстроек модулирующих полей от резонанса мы переходим в область наличия в системе линейной части коэффициента преломления, и соответственно становится возможным создание ЭМИР. Как следует из данных работы [7], в случае

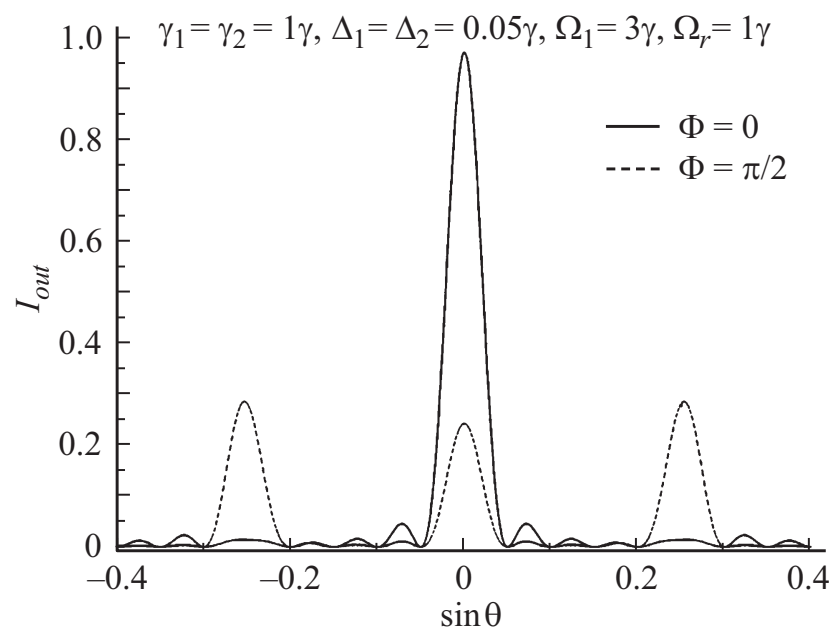

Рис. 3. Зависимость нормированной интенсивности $I_{\text {out }}$ на выходе из среды от $\sin \theta$. Остальные параметры системы следующие: $\gamma_{1}=\gamma_{2}=1 \gamma, \Omega_{1}=3 \gamma, \Omega_{r}=1 \gamma, \Delta_{1}=\Delta_{2}=0.05 \gamma$, $L=7, \Lambda_{x} / \lambda_{2}=0.25, N=5$. 
равенства суммарной фазы $\Phi=0$ в системе возникает эффект КПН, а следовательно, пропадают поглощение и преломление пробного поля, действующего на переходе $|2>-| 3>$, ввиду создания суперпозиционного состояния населенностей нижних уровней $|1>+| 2>$ : взаимодействия между уровнями $|2>-| 3>$ просто нет. Таким образом, не формируется ЭМИР и на выходе из среды не возникает перераспределения интенсивности пробного поля в максимумы первого порядка (рис. 3, кривая $\Phi=0)$.

В случае равенства суммарной фазы $\Phi=\pi / 2$ КПН разрушается [7]. В момент разрушения КПН возникает взаимодействие между уровнями $|2>-| 3>$ и появляется преломление пробного поля $\Omega_{2}$. В результате становится возможным создание ЭМИР. На выходе из среды при этом наблюдается перераспределение интенсивности пробного поля в максимумы первого порядка (рис. 3 , кривая $\Phi=\pi / 2$ ). Проанализировав и подобрав выражения для коэффициентов преломления и поглощения, можно подобрать также параметры для эффективного перераспределения.

В заключение отметим, что предложенная схема помимо новизны обладает внушительным рядом преимуществ: 1) простота реализации и эксплуатации; 2) высокая скорость переключения направления распространения света пробной волны без остановки работы системы; 3) высокая эффективность перераспределения интенсивности поля пробной волны; 4) компактность (подобная ячейка может иметь размер порядка $1 \mathrm{~cm}$ ).

Все перечисленные выше свойства могут вызвать существенный интерес для реализации высокоэффективного быстродействующего переключателя света на основе фазового управления КПН в среде из трехуровневых $\Lambda$-атомов.

\section{Финансирование работы}

Работа поддержана Министерством образования и науки РФ (проект 3.821.2014/K), грантом 074-U01 для лидирующих университетов РФ и грантом Российского фонда фундаментальных исследований 17-02-00598 А.

\section{Конфликт интересов}

Авторы заявляют, что у них нет конфликта интересов.

\section{Список литературы}

[1] Ling H.Y., Li Y., Xiao M. // Phys. Rev. A. 1998. V. 57. N 2. P. $1338-1344$.

[2] Mitsunaga M., Imoto N. // Phys. Rev. A. 1999. V. 59. N 6. P. 4773-4776.

[3] Carvalho S.A., de Araujo L.E.E. // Phys. Rev. A. 2011. V. 83. N 5. P. 053825

[4] Xie B., Cai X., Xiao Z.-H. // Opt. Commun. 2012. V. 285. N 2. P. $133-135$

[5] Brown A.W., Xiao M. // Opt. Lett. 2005. V. 30. N 7. P. 699-701.
[6] Zhao L., Duan W., Yelin S.F. // Phys. Rev. A. 2010. V. 82. N 1. P. 013809.

[7] Kosachiov D.V., Matisov B.G., Rozhdestvensky Yu.V. // J. Phys. B. 1992. V. 25. N 11. P. 2473-2488. 